\title{
Monitoreo de la sucesión de especies vegetales leñosas bajo una plantación de Pinus radiata D. Don, en el parque universitario "Francisco Vivar Castro", provincia de Loja, Ecuador
}

\author{
DOI: $10.46932 / \mathrm{sfjdv} 2 \mathrm{n} 1-075$
}

Received in: November 1st, 2020

Accepted in: December 30th, 2020

\author{
Zhofre Aguirre Mendoza \\ Doctor en Ciencias; Docente-Investigador de la Universidad Nacional de Loja. Loja, Ecuador \\ E-mail: zhofre.aguirre@unl.edu.ec
}

\section{Leonardo González Nivelo}

Ingeniero Forestal, Técnico Docente de la Carrera de Ingeniería Forestal, Universidad Nacional de Loja, Loja, Ecuador

E-mail: leonardo.gonzalez@unl.edu.ec

\author{
Nelson Jaramillo Díaz \\ Ingeniero Forestal, Técnico Docente del Herbario LOJA de la Universidad Nacional de Loja \\ E-mail: Nelson.jaramillo@unl.edu.ec \\ Jaime Peña Tamayo \\ Ingeniero Agrónomo, Técnico Investigador del Herbario LOJA de la Universidad Nacional de Loja \\ E-mail: Jaime.pena@unl.edu.ec
}

\section{RESUMEN}

En los andes del Ecuador existen plantaciones de Pinus radiata y Pinus patula, sembradas entre matorrales, páramos y áreas abiertas; y, se desconoce la sucesión vegetal, sus cambios y desarrollo. Se investigó una plantación de Pinus radiata en la hoya de Loja con el objetivo de caracterizar la composición florística y monitorear la regeneración natural considerando categorías de regeneración: plántulas, brinzales y latizales. Se establecieron 5 parcelas de $20 \mathrm{~m}$ x $20 \mathrm{~m}$, se anidaron 5 subparcelas de $5 \mathrm{~m}$ x $5 \mathrm{~m}$ y 5 subparcelas de $1 \mathrm{~m} \mathrm{x} 1 \mathrm{~m}$. Se midió altura y diámetro basal. Se determinó la sobrevivencia y crecimiento en un periodo de 12 meses; se analizó la influencia de variables ambientales: luminosidad, pendiente, profundidad del suelo horizonte $\mathrm{O}$, sobre la diversidad y abundancia de la regeneración natural. Se identificaron 24 especies, 21 géneros y 22 familias. La mayor diversidad presentan los latizales; las especies con mayor IVI en las tres categorías son: Piper barbatum, Viburnum triphyllum y Frangula granulosa. Los latizales presentan mayor sobrevivencia $(99,37 \%)$, seguida por brinzales $(87,59 \%)$ y plántulas $(82,76 \%)$. El incremento en altura y diámetro es mayor en Frangula granulosa, Clusia latipes, Critoniopsis pycnantha y Alnus acuminata. No existe influencia significativa de los factores ambientales sobre la diversidad y abundancia de la regeneración natural. La regeneración natural encontrada en las condiciones de hábitat de la plantación; demuestra que es factible la recuperación de la vegetación natural y la presencia de un bosque natural cuando desaparezca la plantación forestal.

Palabras claves: regeneración natural, especies leñosas nativas, factores ambientales, plantación de Pino. 


\section{INTRODUCCIÓN}

Los ecosistemas forestales son áreas de interés biológico debido a los recursos que ofrecen para la sustentabilidad económica y productiva de la sociedad; ello ha provocado un aprovechamiento acelerado y no regulado de los recursos forestales (Vásquez, 1995). Producto del extractivismo masivo se ha inducido a un deterioro de la cubierta vegetal, que provoca consecuencias, como la ausencia de vegetación nativa, la conversión de bosques a pequeños relictos o remanentes, la erosión de los suelos, así como la introducción de especies exóticas que suprimen y dominan parte de la vegetación nativa. Estas amenazas producen la alteración o modificación de los procesos dinámicos, la estructura y composición florísticas de la vegetación nativa (Añazco, Morales, Palacios, Vega y Cuesta, 2010).

En los Andes del Ecuador, se han plantado especies exóticas de crecimiento rápido como: Pinus radiata, Pinus patula, Eucalyptus globulus y Eucalyptus saligna, que abarcan una superficie aproximada de 123720 ha. El establecimiento de estas plantaciones se realizó como alternativa para reforestar y manejar cuencas hidrográficas. Sin embargo, los resultados no han sido favorables debido a la falta de monitoreo y manejo; producto de ello, tales especies han provocado fuertes impactos a la biodiversidad de las áreas reforestadas (Voss, Aguirre y Hofstede, 2001), estas especies se han distribuido espacialmente a través de la dispersión de semillas hacia los ecosistemas naturales cercanos, donde compiten con las especies vegetales nativas allí existentes.

En Ecuador las plantaciones de Pinus rdiata fueron implementadas en la década de 1960 (Caranqui, 2017), ante la necesidad de conservar cuencas hidrográficas y obtener ingresos económicos futuros, con la venta de madera. Las especies fueron plantadas en zonas de páramos, matorrales, como linderos y cercas vivas, provocando disminución y desplazamiento de la vegetación nativa, además de modificaciones estructurales y químicas de los suelos (Carrere, 2005).

En el sotobosque de las plantaciones de Pino en Ecuador, que no han recibido prácticas silviculturales, se observa el crecimiento de especies vegetales nativas, con niveles de densidad y diversidad relativamente bajos (León, 2014); según este autor, el sotobosque de estas plantaciones está ocupado principalmente por especies vegetales arbustivas, que son elementales para dar origen a la sucesión del bosque secundario, que marca la diferencia en cuanto a composición florística, estructura y función, donde las plántulas son susceptible a alta mortalidad como resultado de la competencia entre las especies nativas y las especies introducidas.

Esta investigación se cumplió con los objetivos de determinar la composición florística y monitorear el comportamiento sucesional de las especies vegetales leñosas nativas que crecen bajo la plantación de Pinus radiata en el parque universitario "Francisco Vivar Castro". La información que se presenta trata sobre la composición y diversidad florística, parámetros estructurales y crecimiento en 
diámetro y altura como parte del monitoreo de la dinámica de la regeneración natural de las especies vegetales leñosas nativas bajo plantaciones de Pino.

\section{MATERIALES Y MÉTODOS}

\section{1 ÁREA DE ESTUDIO}

La investigación se realizó en el Parque Universitario "Francisco Vivar Castro", ubicado en La Argelia, parroquia San Sebastián, cantón Loja, es propiedad de la Universidad Nacional de Loja, tiene una superficie de 99,2 ha, en un rango altitudinal de 2130 a $2520 \mathrm{msnm}$. Localizado entre las coordenadas UTM: 700592 - 9554223 N, 700970 - 9553139 S - 701309 - 9553171 E, 699961 - 9554049 W

(Figura 1). En el área existen cinco ecosistemas: bosque andino, matorral, páramo antrópico, bosquete mixto de Juglans neotropica Niels, plantaciones de pino (Pinus patula D. Don y Pinus radita D. Don) y Eucalyptus saligna Labill (Aguirre et al., 2016).

Figura 1. Ubicación geográfica del Parque Universitario "Francisco Vivar Castro".

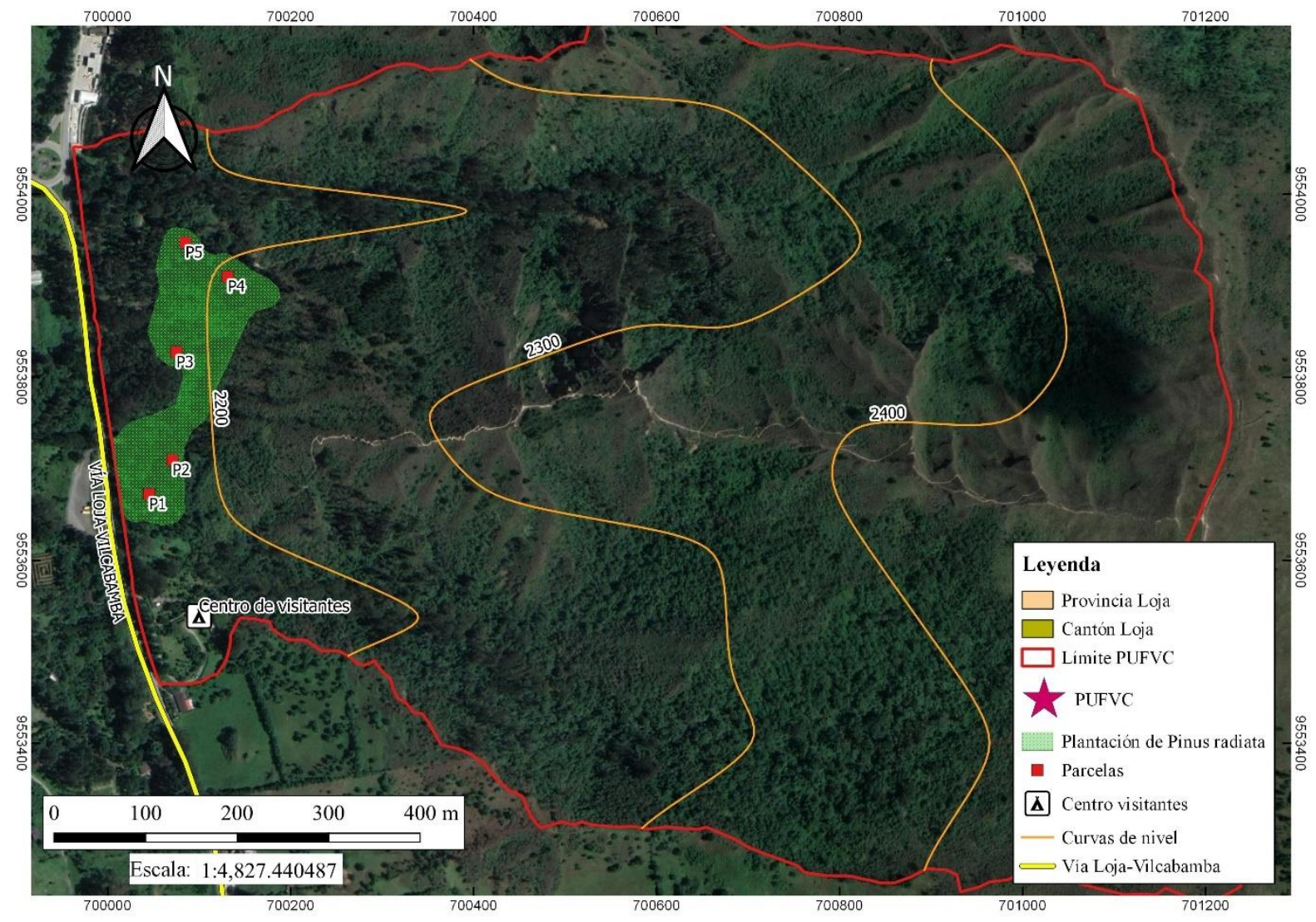




\subsection{UNIDAD DE MUESTREO}

Se aplicó un muestreo sistemático siguiendo la metodología de Aguirre (2019). Para ello, se instaló cinco parcelas permanentes de $20 \mathrm{~m}$ x $20 \mathrm{~m}\left(400 \mathrm{~m}^{2}\right)$, considerando un distanciamiento de $100 \mathrm{~m}$ entre parcelas, el área se delimitó con una cuerda de color rojo; en los extremos de cada parcela se colocó mojones de cemento con un tubo PVC, esto para facilitar el seguimiento y monitoreo (Figura 2).

\subsection{COMPOSICIÓN FLORÍSTICA DE LAS CATEGORÍAS DE REGENERACIÓN}

En las parcelas y subparcelas se identificó y contabilizó la regeneración natural de las especies vegetales leñosas nativas, éstas se etiquetaron con una placa de plástico color amarillo, con un código para cada categoría de regeneración, esto con el fin de realizar el monitoreo de la sobrevivencia, crecimiento diamétrico y altura de las especies (Aguirre, 2019).

\subsection{MONITOREO DEL COMPORTAMIENTO SUCESIONAL DE LAS ESPECIES VEGETALES LEÑOSAS NATIVAS}

Para el registro de la regeneración natural se utilizó las categorías propuestas por Orozco y Brumer (2002) que son: Latizal: individuos > a 1,50 de altura y $\leq$ a $10 \mathrm{~cm}$ de diámetro; Brinzal: individuos > a 30 $\mathrm{cm}$ altura $\mathrm{y} \leq 1,50 \mathrm{~m}$ altura; Plántulas: individuos $\leq 30 \mathrm{~cm}$ altura. Se establecieron 5 parcelas de $20 \mathrm{~m} \mathrm{x}$ $20 \mathrm{~m}$, dentro se anidaron 5 subparcelas de $5 \mathrm{~m}$ x $5 \mathrm{~m}$ y 5 subparcelas de $1 \mathrm{~m}$ x $1 \mathrm{~m}$, respectivamente.

Figura 2. Diseño de las parcelas permanentes, para el levantamiento de información de la regeneración natural de las especies vegetales leñosas nativas.
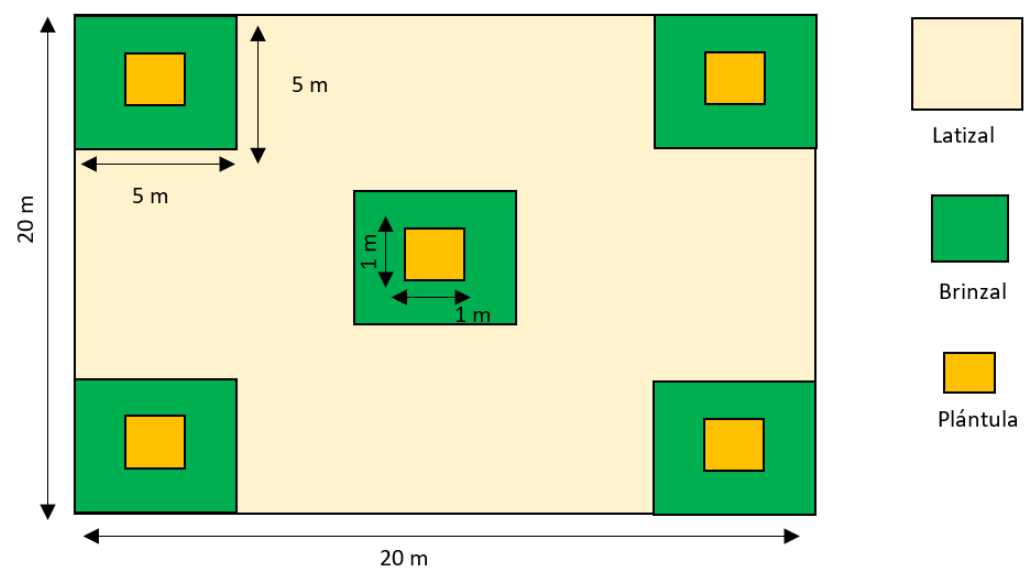

Se colectaron los datos en dos instancias, una al inicio y la segunda a los doce meses de establecidas las parcelas. En la primera fase se identificaron las especies, etiquetó y midió la altura y diámetro basal de los individuos por categoría de regeneración. En la segunda fase se remidió las variables dasométricas, identificación de individuos vivos y muertos. Además, se registro datos de tres variables 
ambientales: luminosidad utilizando una cámara fotográfica CANON 35X IS la cual permitió capturar imágenes del dosel tomando en consideración una altura de 1,30 m desde el suelo en sentido vertical, se realizó en un día nublado para evitar sobrestimaciones de luz (Mostacedo y Fredericksen, 2000), luego se procesaron las fotografías en la aplicación HabitApp (Mejía y Barón, 2019); la pendiente para lo cual se usó un clinómetro (Cruz, 2011); y, la profundidad del suelo horizonte O, utilizando un flexómetro.

\subsection{ANÁLISIS DE DATOS}

Se determinó la composición florística, diversidad y parámetros estructurales de la vegetación: densidad relativa, frecuencia relativa, Índice de Valor de Importancia simplificado para cada categoría de regeneración natural. Para el cálculo de sobrevivencia se consideró el número de individuos vivos y muertos. Y para el incremento de diámetro y altura se promedió los valores iniciales y finales registrados por especies (Tabla 1).

Se utilizaron las especies y su abundancia para establecer la influencia con las variables ambientales (Jirón, 2017). Para el análisis de componentes principales (PCA) se usó el programa estadístico R; además, se realizó un coeficiente de correlación de Pearson con un valor $\alpha<=0,05$ usando el programa estadístico InfoStat 2020.

\begin{tabular}{ll}
\multicolumn{2}{l}{ Tabla 1. Fórmulas para el cálculo de parámetros estructurales y crecimiento en diámetro y altura } \\
$\begin{array}{l}\text { Parámetro } \\
\text { Densidad relativa }(\%)\end{array}$ & $\begin{array}{l}\mathrm{Dr} \%=\frac{\text { número de individuos de la especie }}{\text { total de especies }} * 100 \\
\text { Frecuencia relativa }(\%)\end{array}$ \\
$\begin{array}{l}\text { Índice de valor de importancia } \%=\frac{\text { número de parcelas en la que se inventarea la especie }}{\text { sumatoria de frecuencia de todas las especies }} * 100 \\
(\%)\end{array}$ & $\begin{array}{l}\mathrm{IVI}=\mathrm{Dr}+\mathrm{Fr} \\
\text { Sobrevivencia }(\%)\end{array}$ \\
Incremento diamétrico $(\mathbf{m})$ & $\mathrm{Cr}$ Dobrevivencia $=$ indv vivos $* 100 /$ total indv \\
& $\mathrm{CrH}=H_{f}-H_{i}$
\end{tabular}

\section{RESULTADOS}

\subsection{COMPOSICIÓN FLORÍSTICA GENERAL}

La composición florística de la regeneración natural bajo el dosel de la plantación de Pinus radiata, es de 24 especies pertenecientes a 21 géneros y 22 familias, con un total de 648 individuos. De las 24 especies 14 son árboles y 10 arbustos. Las familias más diversas son Asteraceae, Primulaceae, Melastomataceae y Solanaceae. 


\subsection{COMPOSICION FLORISTICA DE CADA CATEGORÍA DE REGENERACIÓN NATURAL}

La composición florística de las plántulas, es de 5 especies que pertenece a 5 familias. Las especies representativas son: Viburnum triphyllum y Piper barbatum. La composición florística de brinzales es de 17 especies dentro de 14 familias, de las cuales Melastomataceae, Primulaceae y Solanaceae son representativas (Tabla 2). Piper barbatum, Viburnum triphyllum, Frangula granulosa y Oreopanax rosei son las especies más abundantes. La composición florística de latizales es de 21 especies que pertenece a 17 familias, de las cuales Asteraceae, Melastomataceae, Primulaceae y Solanaceae son mas diversas; Piper barbatum, Viburnum triphyllum, Solanum aphyodendron y Frangula granulosa, son las especies más abundantes (Tabla 2).

Tabla 2. Especies vegetales presentes en la categorías de regeneración natural, bajo el dosel de plantación de Pinus radiata del PUFVC, Loja.

\begin{tabular}{|c|c|c|c|c|}
\hline \multirow{2}{*}{ Especie } & \multicolumn{3}{|c|}{ Categorías } & \multirow{2}{*}{$\begin{array}{l}\text { Número de } \\
\text { individuos }\end{array}$} \\
\hline & Plántula & Brinzal & Latizal & \\
\hline Piper barbatum Kunth & 9 & 57 & 184 & 250 \\
\hline Viburnum triphyllum Benth. & 10 & 30 & 132 & 172 \\
\hline Solanum aphyodendron S. Knapp & & 8 & 42 & 50 \\
\hline Frangula granulosa (Ruiz \& Pav.) Grubov & 4 & 11 & 37 & 52 \\
\hline Oreopanax rosei Harms & 2 & 11 & 13 & 26 \\
\hline Myrsine andina (Mez) Pipoly & & 5 & 10 & 15 \\
\hline Solanum cf. cutervanum Zahlbr. & & 4 & 9 & 13 \\
\hline Tournefortia fuliginosa Kunth & & & 7 & 7 \\
\hline Myrsine sodiroana (Mez) Pipoly & 4 & 3 & 6 & 13 \\
\hline Palicourea anceps Standl. & & 3 & 6 & 9 \\
\hline Verbesina cf. arborea Kunth & & & 6 & 6 \\
\hline Critoniopsis pycnantha (Benth.) H.Rob. & & 1 & 4 & 5 \\
\hline Siparuna muricata (Ruiz \& Pav.) A. DC. & & 1 & 4 & 5 \\
\hline Miconia lutescens (Bonpl.) DC. & & 3 & 3 & 6 \\
\hline Miconia obscura (Bonpl.) Naudin & & 2 & 3 & 5 \\
\hline Cedrela montana Moritz ex Turcz. & & & 2 & 2 \\
\hline Roupala monosperma (Ruiz \& Pav.) I.M.Johnst. & & & 2 & 2 \\
\hline Alnus acuminata Kunth & & & 1 & 1 \\
\hline Chionanthus pubescens Kunth & & & 1 & 1 \\
\hline Clusia latipes Planch. \& Triana & & 1 & 1 & 2 \\
\hline Mauria heterophylla Kunth & & & 1 & 1 \\
\hline Clethra fimbriata Kunth & & 1 & & 1 \\
\hline $\begin{array}{l}\text { Morella pubescens (Humb. \& Bonpl. ex Willd.) } \\
\text { Wilbur }\end{array}$ & & 1 & & 1 \\
\hline Saurauia bullosa Wawra & & 3 & & 3 \\
\hline Total & 29 & 145 & 474 & 648 \\
\hline
\end{tabular}




\subsection{PARÁMETROS ESTRUCTURALES DE LA REGENERACIÓN NATURAL}

En la Tabla 3 se presenntan los parámetros estructuraes de la categoría de plántula, las especies con mayor IVI son: Piper barbatum, Viburnum triphyllum y Frangula granulosa

Tabla 3. Parámetros estructurales en la categoría plántulas bajo la plantación de Pinus radiata del PUFVC, Loja.

Piper barbatum Kunth

Viburnum triphyllum Benth.

Frangula granulosa (Ruiz \& Pav.) Grubov

Oreopanax rosei Harms

Myrsine sodiroana (Mez) Pipoly
Familia

Piperaceae

Adoxaceae

Rhamnaceae

Araliaceae

Primulaceae
FR (\%)

30,00

20,00

20,00

20,00

10,00
DR (\%)

31,03

34,48

13,79

6,90

13,79
IVI $(\%)$

30,52

27,24

16,90

13,45

11,90

$\mathrm{FR}=$ Frecuencia Relativa , DR = Densidad Relativa, IVI = Índice Valor Importancia

En la categoría de brinzal, las especies con mayor IVI son: Piper barbatum, Viburnum triphyllum, Oreopanax rosei y Frangula granulosa (Tabla 4).

Tabla 4. Parámetros estructurales en la categoría de brinzal, bajo la plantación de Pinus radiata del PUFVC, Loja.

\section{Piper barbatum Kunth \\ Viburnum triphyllum Benth. \\ Oreopanax rosei Harms}

Frangula granulosa (Ruiz \& Pav.) Grubov

Solanum aphyodendron S. Knapp

Solanum cf. cutervanum Zahlbr.

Miconia lutescens (Bonpl.) DC.

Myrsine sodiroana (Mez) Pipoly

Myrsine andina (Mez) Pipoly

Palicourea anceps Standl.

Saurauia bullosa Wawra

Miconia obscura (Bonpl.) Naudin

Clethra fimbriata Kunth

Clusia latipes Planch. \& Triana

Critoniopsis pycnantha (Benth.) H.Rob.

Morella pubescens (Humb. \& Bonpl. ex Willd.)

Wilbur

Siparuna muricata (Ruiz \& Pav.) A. DC.

\section{Familia}

Piperaceae

Adoxaceae

Araliaceae

Rhamnaceae

Solanaceae

Solanaceae

Melastomataceae

Primulaceae

Primulaceae

Rubiaceae

Actinidaceae

Melastomataceae

Clethraceae

Clusiaceae

Asteraceae

Myricaceae

Siparunaceae
FR $(\%)$

10,91

10,91

10,91

7,27

9,09

7,27

5,45

5,45

3,64

3,64

3,64

3,64

3,64

3,64

3,64

3,64

3,64
DR $(\%)$

39,31

20,69

7,59

7,59

5,52

2,76

2,07

2,07

3,45

2,07

2,07

1,38

0,69

0,69

0,69

0,69

0,69

IVI (\%)

25,11

15,80

9,25

7,43

7,30

5,02

3,76

3,76

3,54

2,85

2,85

2,51

2,16

2,16

2,16

2,16

2,16

FR $=$ Frecuencia Relativa $;$ DR $=$ Densidad Relativa, IVI = Índice Valor Importancia

Las especies con mayor IVI en la categoría Latizal son: Piper barbatum, Viburnum thriphyllum, Solanum aphyodendron y Frangula granulosa (Tabla 5).

Tabla 5. Parámetros estructurales en la categoría de latizal, bajo la plantación de Pinus radiata del PUFVC, Loja.

Piper barbatum Kunth

Viburnum triphyllum Benth.

Solanum aphyodendron S. Knapp

Frangula granulosa (Ruiz \& Pav.) Grubov

Oreopanax rosei Harms

Solanum cf. cutervanum Zahlbr.

Verbesina cf. arborea Kunth
Familia

Piperaceae

Adoxaceae

Solanaceae

Rhamnaceae

Araliaceae

Solanaceae

Asteraceae

\section{FR (\%)}

9,09

9,09

DR (\%)

38,82

27,85

8,86

9,09

7,81

2,74

1,90

1,27
IVI (\%)

23,95

18,47

8,98

8,45

5,92

4,59

4,27 
Critoniopsis pycnantha (Benth.) H.Rob.

Miconia lutescens (Bonpl.) DC.

Tournefortia fuliginosa Kunth

Myrsine sodiroana (Mez) Pipoly

Siparuna muricata (Ruiz \& Pav.) A. DC.

Cedrela montana Moritz ex Turcz.

Myrsine andina (Mez) Pipoly

Palicourea anceps Standl.

Miconia obscura (Bonpl.) Naudin

Roupala monosperma (Ruiz \& Pav.) I.M.Johnst.

Alnus acuminata Kunth

Chionanthus pubescens Kunth

Clusia latipes Planch. \& Triana

Mauria heterophylla Kunth

$\begin{array}{ll}\text { Asteraceae } & 5,45 \\ \text { Melastomataceae } & 5,45 \\ \text { Boraginaceae } & 3,64 \\ \text { Primulaceae } & 3,64 \\ \text { Siparunaceae } & 3,64 \\ \text { Meliaceae } & 3,64 \\ \text { Primulaceae } & 1,82 \\ \text { Rubiaceae } & 1,82 \\ \text { Melastomataceae } & 1,82 \\ \text { Proteaceae } & 1,82 \\ \text { Betulaceae } & 1,82 \\ \text { Oleaceae } & 1,82 \\ \text { Clusiaceae } & 1,82 \\ \text { Anacardiaceae } & 1,82\end{array}$

$0,84 \quad 3,15$

$0,63 \quad 3,04$

$1,48 \quad 2,56$

$1,27 \quad 2,45$

$0,84 \quad 2,24$

$0,42 \quad 2,03$

$2,11 \quad 1,96$

$1,27 \quad 1,54$

$0,63 \quad 1,23$

$0,42 \quad 1,12$

$0,21 \quad 1,01$

$0,21 \quad 1,01$

$0,21 \quad 1,01$

$0,21 \quad 1,01$

FR = Frecuencia Relativa; DR = Densidad Relativa, IVI = Índice Valor Importancia

\subsection{SOBREVIVENCIA DE LA REGENERACIÓN NATURAL}

En la categoría plántula Frangula granulosa y Oreopanax rosei presentan la mayor sobrevivencia con $82,76 \%$; del total de 29 individuos, 5 murieron, 24 se mantuvieron vivos. En la categoría de brinzal 10 especies mantienen el $100 \%$ de vitalidad en sus individuos, que pertenecen a Clethra fimbriata, Clusia latipes, Critoniopsis pycnantha, Myrsine andina. De un total de 145 individuos, 18 están muertos y 127 se mantuvieron vivos, dando como resultado 87,59\% de sobrevivencia para esta categoría. En la categoría latizal presentan mayor sobrevivencia Alnus acuminata, Cedrela montana, Chionanthus pubescens, Clusia latipes, Critoniopsis pycnantha; De 474 individuos, 471 se mantuvieron vivos dando un porcentaje de sobrevivencia de 99,37\% (Tabla 6).

Tabla 6. Análisis de sobrevivencia en la categoría de plántula, brinzal y latizal bajo la plantación de Pinus radiata del PUFVC, Loja

\begin{tabular}{|c|c|c|c|c|}
\hline \multirow{3}{*}{ Especie } & \multirow{3}{*}{ Familia } & \multicolumn{3}{|c|}{ Sobrevivencia $(\%)$} \\
\hline & & & ategoría & \\
\hline & & Plántula & Brinzal & Latizal \\
\hline Alnus acuminata Kunth & Betulaceae & & & 100,00 \\
\hline Cedrela montana Moritz ex Turcz. & Meliaceae & & & 100,00 \\
\hline Chionanthus pubescens Kunth & Oleaceae & & & 100,00 \\
\hline Clethra fimbriata Kunth & Clethraceae & & 100,00 & \\
\hline Clusia latipes Planch. \& Triana & Clusiaceae & & 100,00 & 100,00 \\
\hline Critoniopsis pycnantha (Benth.) H.Rob. & Asteraceae & & 100,00 & 100,00 \\
\hline Frangula granulosa (Ruiz \& Pav.) Grubov & Rhamnaceae & 100,00 & 81,82 & 100,00 \\
\hline Mauria heterophylla Kunth & Anacardiaceae & & & 100,00 \\
\hline Miconia lutescens (Bonpl.) DC. & Melastomataceae & & 66,67 & 100,00 \\
\hline Miconia obscura (Bonpl.) Naudin & Melastomataceae & & 100,00 & 100,00 \\
\hline $\begin{array}{l}\text { Morella pubescens (Humb. \& Bonpl. ex } \\
\text { Willd.) Wilbur }\end{array}$ & Myricaceae & & 100,00 & \\
\hline Myrsine andina (Mez) Pipoly & Primulaceae & & 100,00 & 100,00 \\
\hline Myrsine sodiroana $(\mathrm{Mez})$ Pipoly & Primulaceae & 50,00 & 100,00 & 100,00 \\
\hline Oreopanax rosei Harms & Araliaceae & 100,00 & 90,91 & 92,31 \\
\hline
\end{tabular}


Palicourea anceps Standl. Piper barbatum Kunth Roupala monosperma (Ruiz \& Pav.) I.M.Johnst.

Saurauia bullosa Wawra

Siparuna muricata (Ruiz \& Pav.) A. DC. Solanum aphyodendron S. Knapp Solanum cf. cutervanum Zahlbr. Tournefortia fuliginosa Kunth Verbesina cf. arborea Kunth Viburnum triphyllum Benth.
Rubiaceae

Piperaceae

Proteaceae

Actinidaceae

Siparunaceae

Solanaceae

Solanaceae

Boraginaceae

Asteraceae

Adoxaceae
$66,67 \quad 100,00$

$88,89 \quad 84,21 \quad 99,46$

100,00

100,00

$100,00 \quad 100,00$

$87,50 \quad 97,62$

$100,00 \quad 100,00$

100,00

100,00

$\begin{array}{rrr}80,00 & 90,00 & 100,00 \\ \mathbf{8 2 , 7 6} & \mathbf{8 7 , 5 9} & \mathbf{9 9 , 3 7}\end{array}$

\subsection{INCREMENTO DIAMÉTRICO Y EN ALTURA DE LA REGENERACIÓN NATURAL}

En la categoría plántulas presenta mayor incremento diamétrico Frangula granulosa y Piper barbatum. En cuanto en altura destacan Frangula granulosa, Viburnum triphyllum y Oreopanax rosei con los mayores incrementos (Figura 3)

Figura 3. Incremento diamétrico y altura de plántulas bajo la plantación de Pinus radiata del PUFVC, Loja.
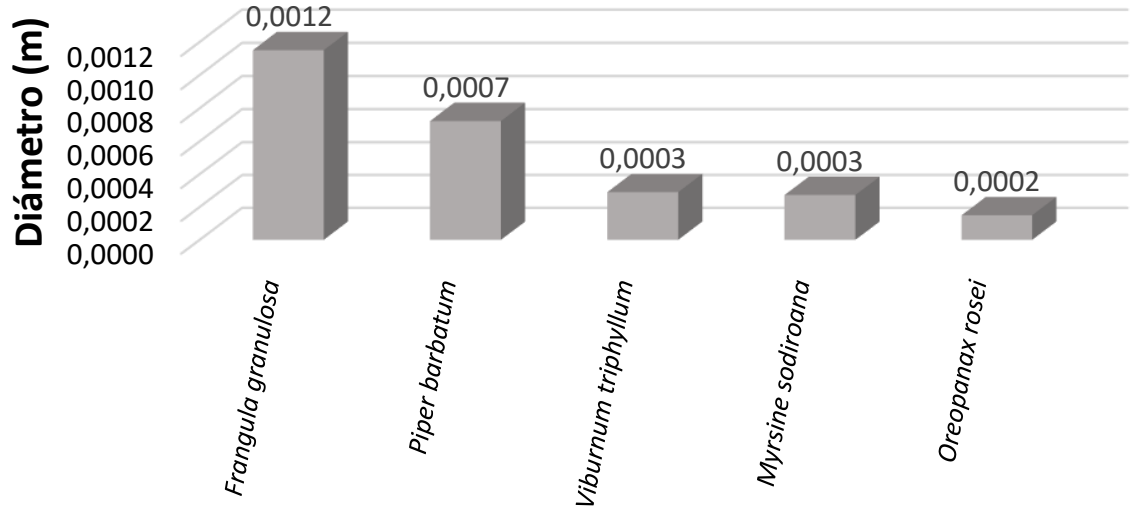

\section{Especies}




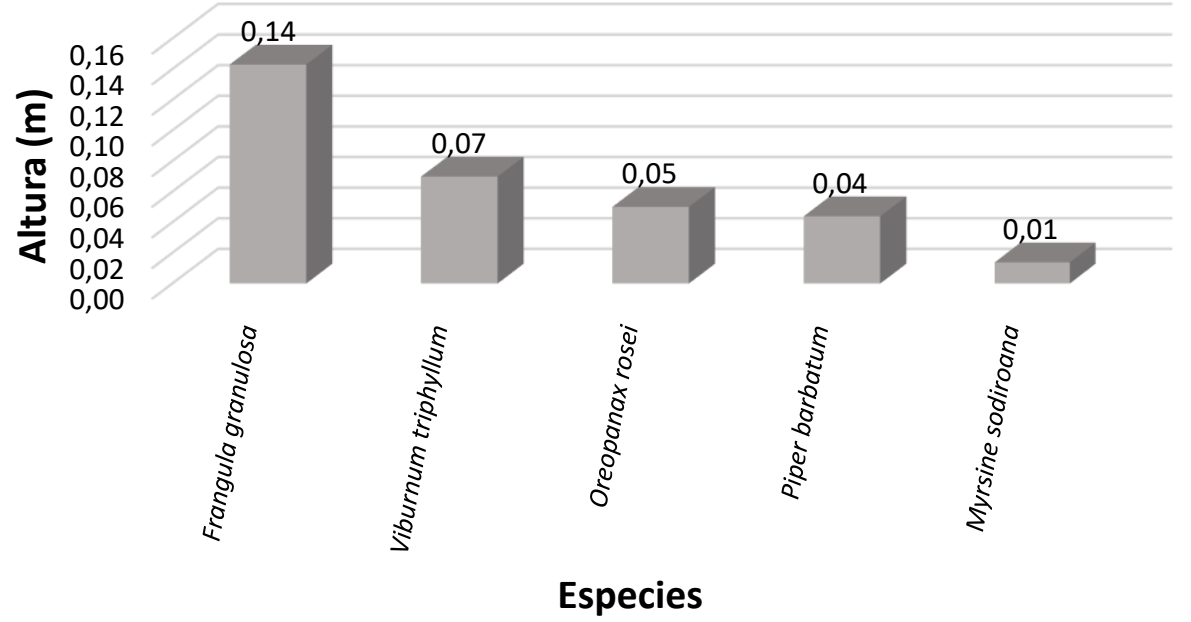

En la Figura 4, se muestran las 10 especies de la categoría brinzal, que tienen mayor incremento en diámetro Clusia latipes y Solanum cf. cutervanum. El mayor incremento en altura presentan Critoniopsis pycnantha, Clethra fimbriata, Siparuna muricata y Solanum aphyodendron.

Figura 4. Incremento diamétrico y altura de brinzales bajo la plantación de Pinus radiata del PUFVC, Loja.
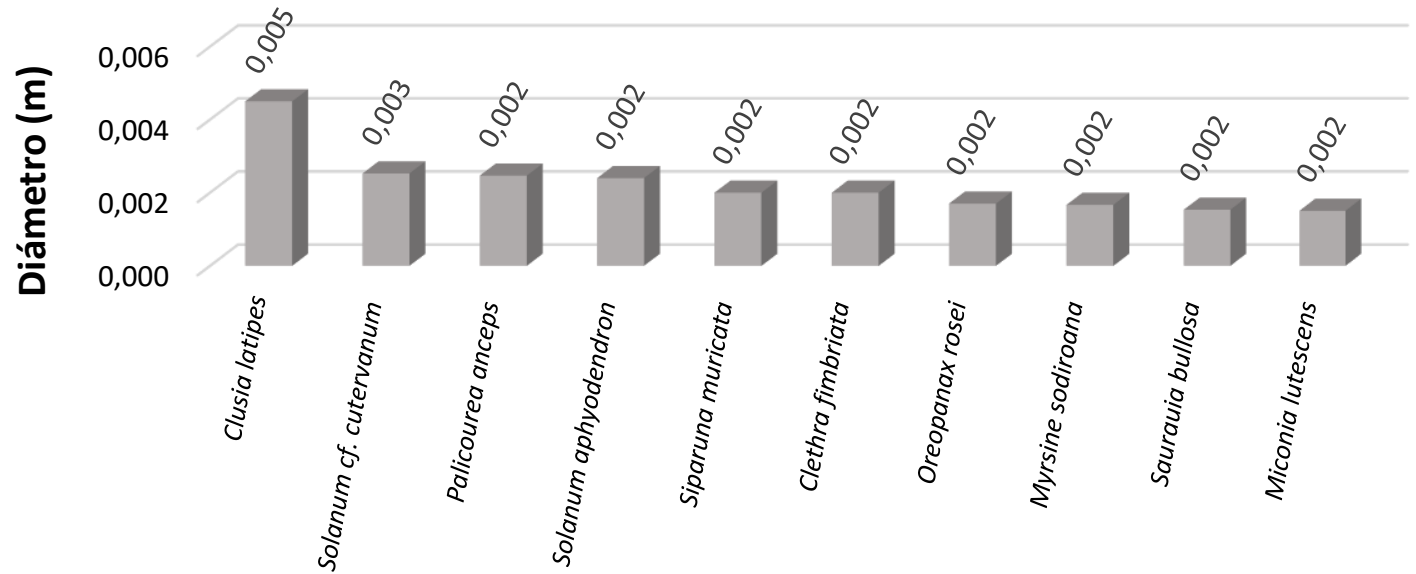

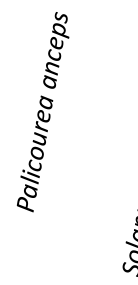

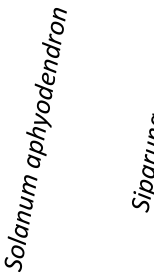

离

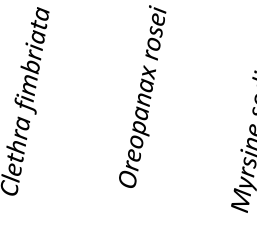

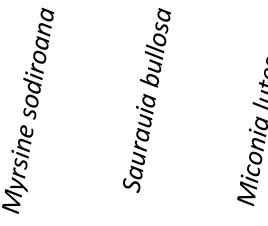

\section{Especies}




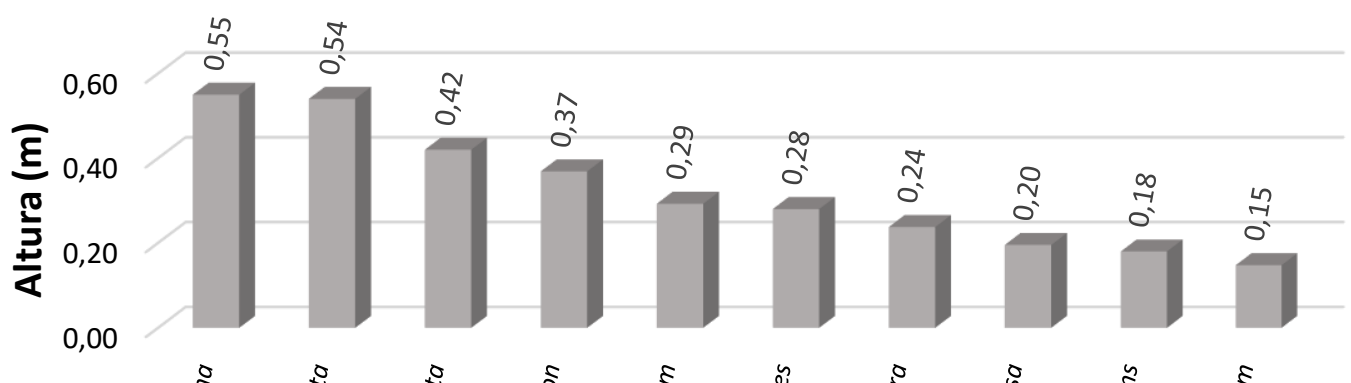

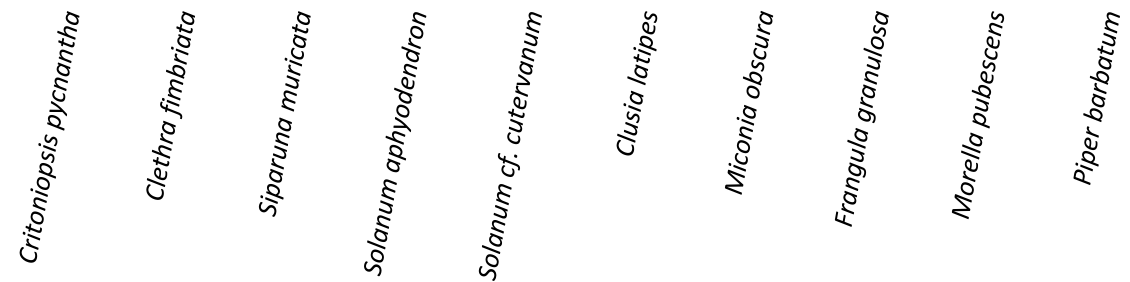

Especies

En los latizales las 10 especies con mayor incremento diamétrico son Alnus acuminata y Cedrela montana. Y las especies con mayor incremento en altura son: Alnus acuminata, Roupala monosperma, Viburnum triphyllum, Frangula granulosa y Miconia lutescens (Figura 5).

Figura 5. Incremento diamétrico y altura de latizales bajo la plantación de Pinus radiata del PUFVC, Loja.

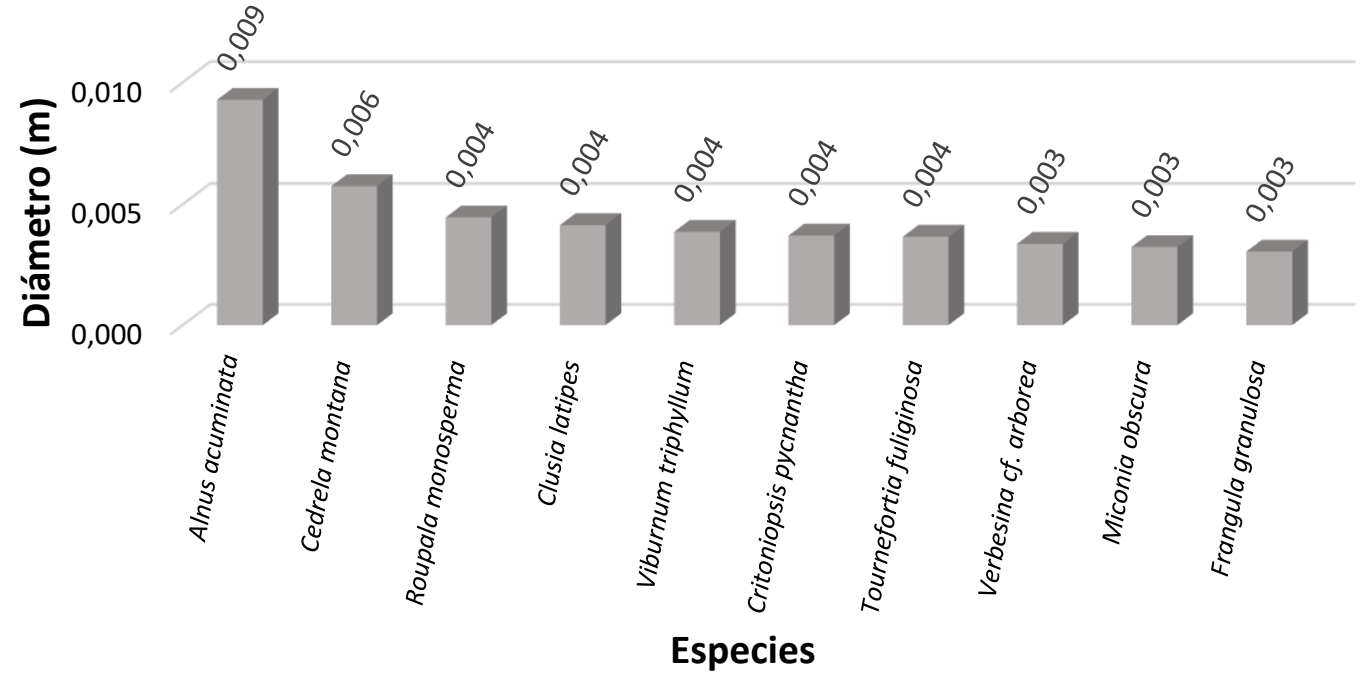




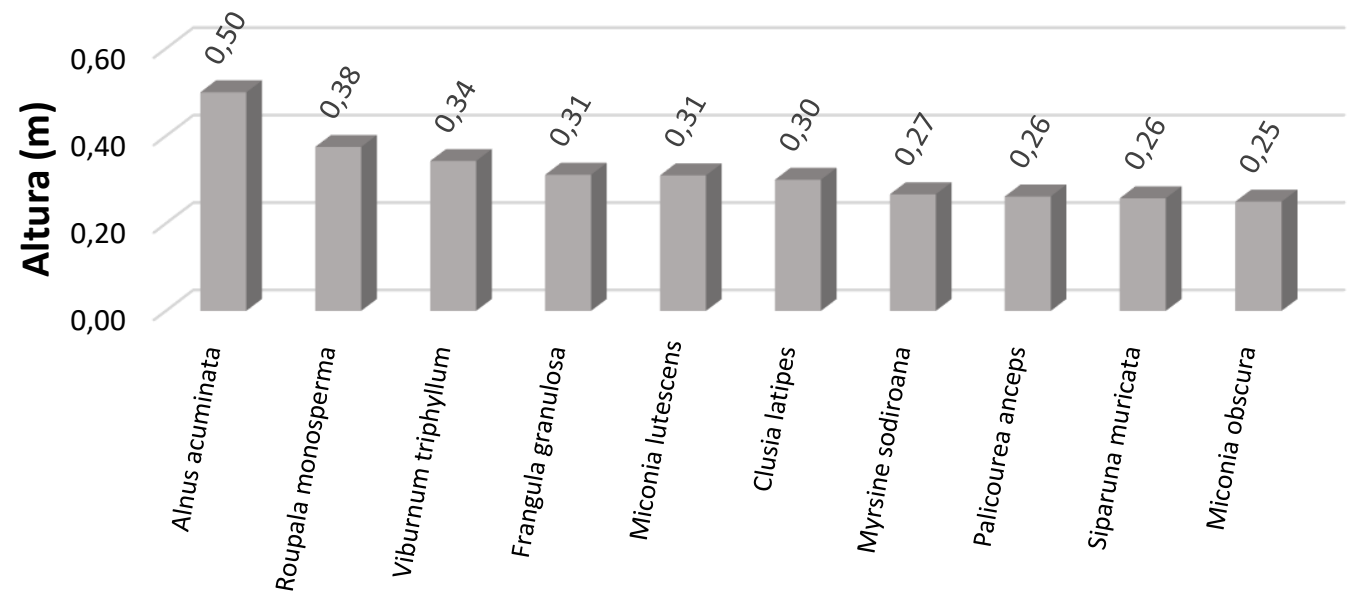

Especies

\subsection{INFLUENCIA DE LAS VARIABLES SOBRE LA REGENERACIÓN NATURAL}

En la categoría de plántulas existe correlación entre el número de especies vs número de individuos $(\mathrm{r}=0,94 \mathrm{p}=0,00)$; y se evidencia correlación moderadamente débil y no significativa de la luminosidad sobre el número de especies $(\mathrm{r}=0,31 \mathrm{p}=0,13)$ e individuos $(\mathrm{r}=0,21 \mathrm{p}=0,32)$. La variable con baja correlación es la pendiente vs número de especies e individuos presentando valores de $\mathrm{r}=0,10 \mathrm{p}=0,64 \mathrm{y}$ $\mathrm{r}=0,08 \mathrm{p}=0,70$ respectivamente. Además, cabe mencionar que la variable profundidad del horizonte $\mathrm{O}$ $(\mathrm{r}=-0,36 \mathrm{p}=0,08 \mathrm{y}-0,26 \mathrm{p}=0,21)$ presentan una correlación negativa, poco significativa (Tabla 7).

Tabla 7. Correlación de Pearson de las variables ambientales en plántulas de la plantación de Pinus radiata del PUFVC

\begin{tabular}{|c|c|c|c|c|c|}
\hline Variables & Especies & Individuos & $\begin{array}{l}\text { Profundidad } \\
\text { horizonte } \mathrm{O}\end{array}$ & Pendiente & Luminosidad \\
\hline Especies & 1,00 & 0,00 & 0,21 & 0,64 & 0,13 \\
\hline Individuos & 0,94 & 1,00 & 0,08 & 0,70 & 0,32 \\
\hline $\begin{array}{l}\text { Profundidad } \\
\text { horizonte } O\end{array}$ & $-0,26$ & $-0,36$ & 1,00 & 0,45 & 0,42 \\
\hline Pendiente & 0,10 & 0,08 & $-0,16$ & 1,00 & 0,74 \\
\hline Luminosidad & 0,31 & 0,21 & 0,17 & $-0,07$ & 1,00 \\
\hline
\end{tabular}

El análisis de componentes principales (PCA) indica que en la Dim1 (44\%) existe mayor explicación de los datos y en la Dim2 $(25,20 \%)$ es menor, dando un potencial explicativo de 69,20\%. Los ángulos $\left(<90^{\circ}\right)$ de formación entre vectores indican que el número de individuos y especies se correlacionan fuertemente, además, la pendiente y la luminosidad tienen buena correlación con el número de individuos y especies. El ángulo $\left(>90^{\circ}\right)$ formado entre los vectores pendiente, profundidad horizonte O y luminosidad no presentan correlación positiva. La mayor variabilidad de datos se representa en el número de individuos y especies (Figura 6). 
Figura 6. PCA - Biplot de variables ambientales en la categoría de plántulas, bajo plantación de Pinus radiata.

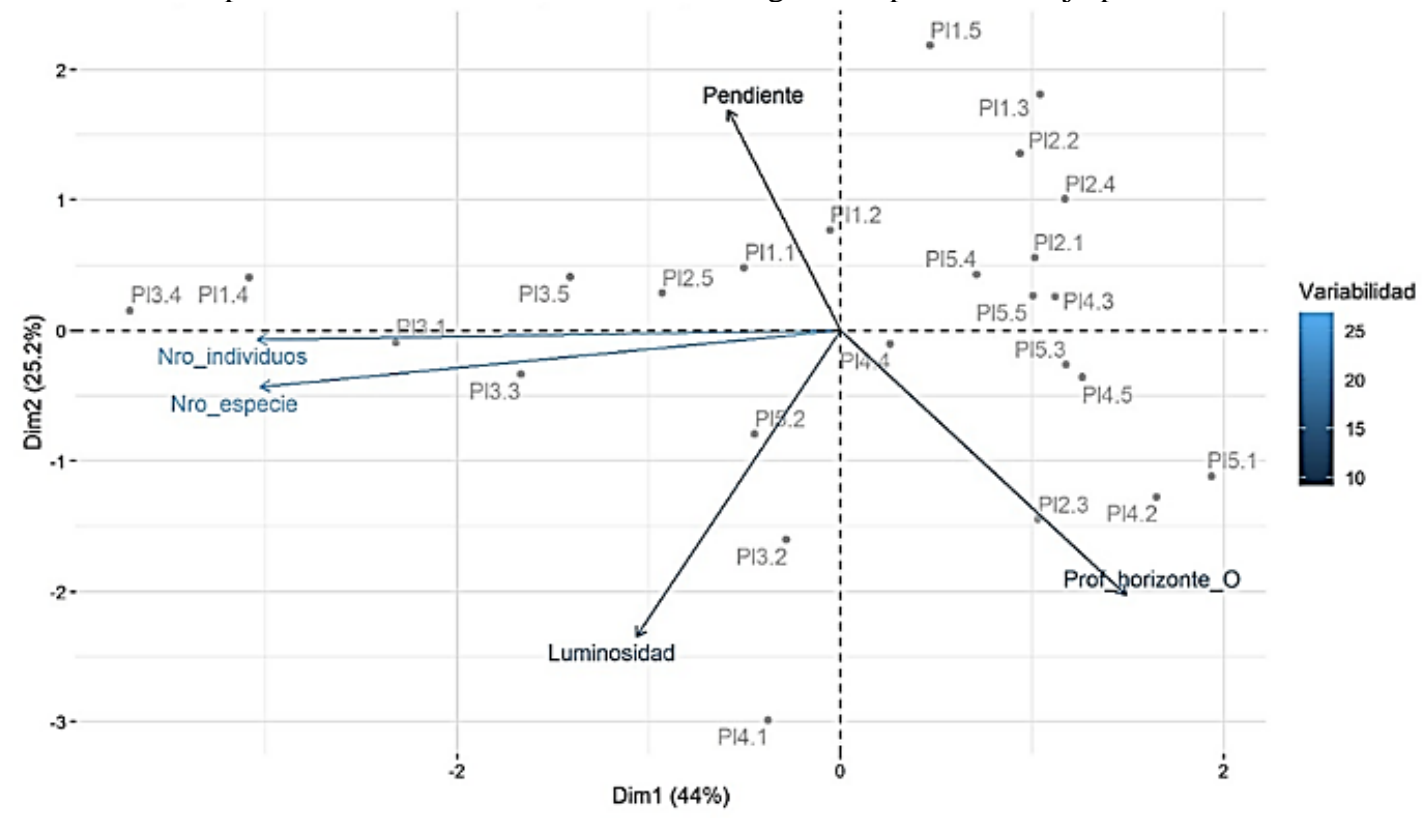

La correlación de Pearson en brinzales muestra que las especies vs individuos tienen fuerte correlación y significancia $(\mathrm{r}=0,85 \mathrm{p}=0,00)$, posterior a ello la profundidad horizonte $\mathrm{O}$ tiene débil correlación y significancia $(r=0,25 \mathrm{p}=0,22)$, la pendiente con una correlación débil $(\mathrm{r}=0,17 \mathrm{p}=0,42)$, las cuales presentan una disminución gradual de correlación con las especies. La pendiente $(r=0,06$ p = 0,79) tiene correlación casi nula con el número de individuos en esta categoría. Existe correlación negativa y moderadamente débil de la luminosidad $(\mathrm{r}=-0,30 \mathrm{p}=0,14 ;-0,35 \mathrm{p}=0,09 \mathrm{y}-0,07 \mathrm{p}=0,74)$ (Tabla 8).

Tabla 8. Correlación de Pearson de las variables ambientales en brinzales de la plantación de Pinus radiata del PUFVC.

\begin{tabular}{lccccc}
\multicolumn{1}{c}{ Variables } & Especies & Individuos & $\begin{array}{c}\text { Profundidad } \\
\text { horizonte }\end{array}$ & Pendiente & $\begin{array}{c}\text { Luminosidad } \\
\text { Especies }\end{array}$ \\
$\begin{array}{l}\text { Individuos } \\
\text { Profundidad }\end{array}$ & 1,00 & 0,00 & 0,22 & 0,42 & 0,14 \\
horizonte O & 0,85 & 1,00 & 0,94 & 0,79 & 0,09 \\
Pendiente & 0,25 & 0,02 & 1,00 & 0,45 & 0,42 \\
Luminosidad & 0,17 & 0,06 & $-0,16$ & 1,00 & 0,74 \\
& $-0,30$ & $-0,35$ & 0,17 & $-0,07$ & 1,00
\end{tabular}

En el PCA se evidencia que la Dim1 (41,70 \%) aporta con la mayor explicación de datos y por otra parte la Dim1 $(24,80 \%)$ tiene un aporte menor, dando un potencial explicativo del 66,50 \%. En esta categoría de regeneración los vectores de pendiente y profundidad horizonte $\mathrm{O}$ son influyentes y se correlacionan positivamente en mayor porcentaje con el número de individuos y especies. El vector luminosidad esta opuesto a la pendiente, número de individuos y especies, lo cual indica que existe fuerte correlación negativa. Cabe mencionar que al igual que la categoría de plántulas se evidencia mayor variabilidad de datos en el número de individuos y especies (Figura 7). 
Figura 7. PCA - Biplot de variables ambientales en la categoría de brinzales, bajo plantación de Pinus radiata.

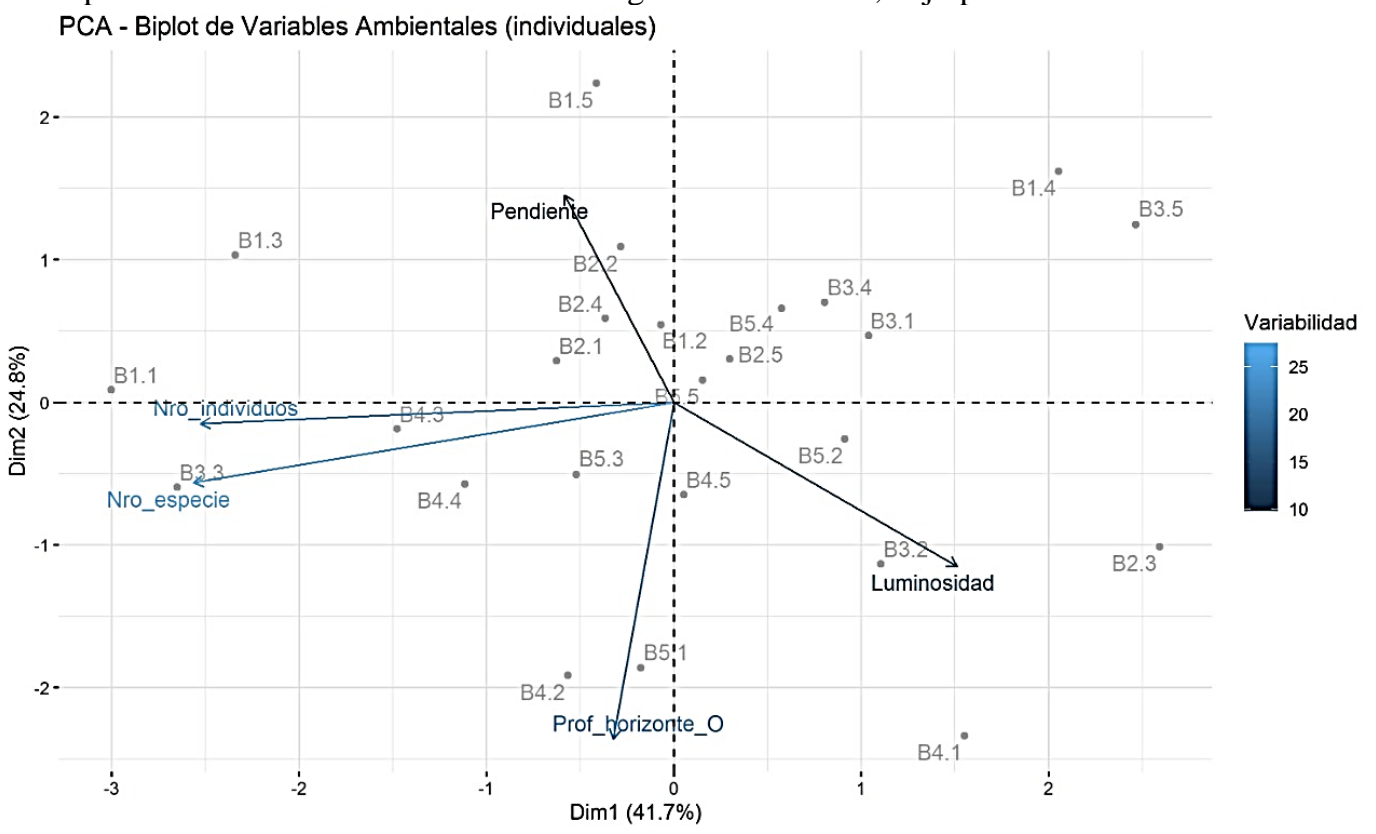

La correlación de Pearson demuestra que para latizales la variable con fuerte correlación y significancia se da entre especies e individuos $(\mathrm{r}=0,61 \mathrm{p}=0,28)$. Además, la profundidad horizonte $\mathrm{O}(\mathrm{r}=$ $0,55 \mathrm{p}=0,34)$, pendiente $(\mathrm{r}=0,49 \mathrm{p}=0,40)$ y luminosidad $(\mathrm{r}=0,48 \mathrm{p}=0,41)$ están moderadamente correlacionadas con las especies. La variable luminosidad disminuye gradualmente con la profundidad horizonte $\mathrm{O}(\mathrm{r}=0,33 \mathrm{p}=0,58)$ e individuos $(\mathrm{r}=0,06 \mathrm{p}=0,92)$ presentando correlación moderadamente débil a débil. Sin embargo, existe correlación negativa casi nula de la pendiente con los individuos $(r=-0,03$ $\mathrm{p}=0,96)$ y la profundidad negativa moderadamente débil horizonte $\mathrm{O}(\mathrm{r}=-0,30 \mathrm{p}=0,62)$ (Tabla 9).

Tabla 9. Correlación de Pearson de las variables ambientales en latizales de la plantación de Pinus radiata del PUFVC.

$$
\text { Variables }
$$

\section{Especies}

Individuos

Profundidad

horizonte $\mathbf{O}$

Pendiente

Luminosidad

\section{Especies}

1,00

0,61

0,55

0,49

0,48
Pendiente

0,40

0,96

0,62

1,00

$-0,27$
Luminosidad

0,41

0,92

0,58

0,66

1,00

En los latizales se evidencia que los datos tienen mayor explicación por la Dim1 (50,08 \%) y menor en la Dim2 (28,20\%). El vector que presenta la influencia más alta es la profundidad horizonte O sobre el número de individuos, seguido por la luminosidad y número de especies que además mantienen una correlación positiva. Sin embargo, la pendiente no genera mayor influencia sobre las variables y su correlación es media con el número de especies. Por otra parte, se distingue mayor variabilidad de datos en la profundidad del horizonte $\mathrm{O}$ y el número de especies (Figura 8). 
Figura 8. PCA - Biplot de variables ambientales en la categoría de latizales, bajo plantación de Pinus radiata. PCA - Biplot de Variables Ambientales (individuales)

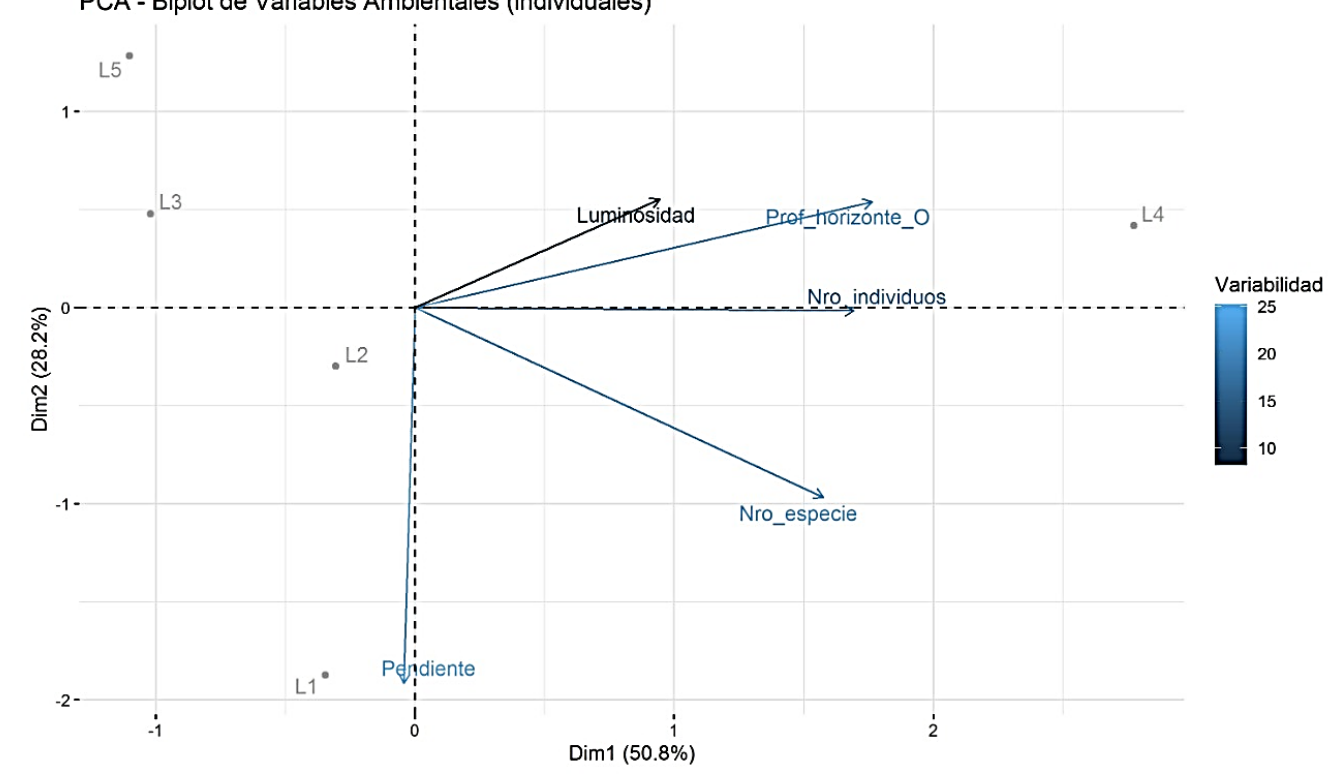

\section{DISCUSIÓN}

\subsection{COMPOSICIÓN FLORÍSTICA DE LAS CATEGORÍAS DE REGENERACIÓN NATURAL EN} LA SUCESIÓN DE ESPECIES VEGETALES LEÑOSAS NATIVAS BAJO EL DOSEL DE LA PLANTACIÓN DE PINUS RADIATA D. DON

La composición florística bajo el dosel de Pinus radiata, presenta 24 especies, dato inferior a lo reportado por León (2014), bajo una plantación de Pinus patula donde registró 28 especies; mientras que Caranqui (2017), en su estudio sobre la regeneración natural luego de la explotación de pino en el sector Tamboloma-Tungurahua, muestra valores superiores con 53 especies, 47 géneros en 25 familias. La diversidad de especies en brinzales (17) y latizales (21) están fuertemente relacionadas, a diferencia de la baja diversidad de plántulas (5 especies), esto es superior a la diversidad registrada por Cavelier y Santos (1999) bajo Pinus radiata que reporta 3 especies, que es bajo en comparación a la diversidad encontrada en la plantación del PUFVC. En este estudio se intuye que la baja diversidad es debido a los procesos antrópicos como incendios forestales, introducción de especies exóticas (pino y eucalipto) que por efecto de alelopatía o condiciones ambientales inhiben la aparición y desarrollo de grupos de especies arbustivas o arbóreas.

Las familias más diversas registradas en el componente leñoso bajo la plantación de Pinus radiata son: Primulaceae, Asteraceae, Solanaceae y Melastomataceae; estos resultados que se asemejan a los reportados por Aguirre, Díaz, Muñoz y Muñoz (2019) en una investigación bajo plantación de Pinus radiata en el PUFV. Datos similares a este estudio recalca Franco, Betancur y Franco (2010) en un remanente de bosque andino donde las especies pertenecen principalmente a las familias Asteraceae, Solanaceae y Melastomataceae. 
4.2 PARÁMETROS ESTRUCTURALES POR CATEGORÍA DE REGENERACIÓN NATURAL EN LA SUCESIÓN DE ESPECIES VEGETALES LEÑOSAS NATIVAS BAJO EL DOSEL DE LA PLANTACIÓN DE PINUS RADIATA D. DON

Las especies con mayor índice valor de importancia son: Piper barbatum y Viburnum triphyllum en las tres categorías de regeneración natural, estos datos se asemejan a los reportados por Aguirre, Díaz, Muñoz y Muñoz (2019), quienes determinaron la sucesión natural bajo Pinus radiata y demostraron que la importancia ecológica de las especies arbustivas se ven representadas por Piper bogotense y Viburnum triphyllum. Además, León (2014), en un estudio sobre la influencia de la luz en la regeneración natural de especies leñosas bajo plantaciones de Pinus patula evidenció mayor abundancia de Piper bogotense (332 ind), lo cual es congruente con este estudio, donde se obtuvo un registro 250 individuos de Piper barbatum.

Las categorías de regeneración plántula, brinzal y latizal, presentan una abundancia que se incrementa respectivamente en individuos (29, 145 y 474); cabe destacar que Jadán et al. (2019) en la investigación de regeneración bajo ecosistemas naturales y plantaciones de Pinus patula en Azuay reporta 759 individuos, valores casi similares a los de esta investigación. Contrario a lo mencionado por Aguirre, Celi y Herrera (2018) en un bosque siempreverde montano bajo registran que los brinzales presentan alta regeneración (14 167 ind/ha), seguido de plántulas, mientras que los latizales presentan bajos niveles. Esto genera la importancia del estudio de las especies arbóreas leñosas, debido a que son biotipos perennes que presentan mayor dificultad para germinar y desarrollarse frente a plantas herbáceas

\subsection{SOBREVIVENCIA POR CATEGORÍA DE REGENERACIÓN NATURAL EN LA SUCESIÓN DE ESPECIES VEGETALES LEÑOSAS NATIVAS BAJO EL DOSEL DE LA PLANTACIÓN DE PINUS} RADIATA D. DON

La sobrevivencia de plántulas es baja, se puede evidenciar especies con hasta $50 \%$, sin embargo, de forma general, está sobre el $80 \%$. Las especies identificadas en esta categoría se encuentran bajo condiciones de supresión con hojarasca, cobertura de dosel y dominadas por brinzales y latizales, por ende, limita el paso de luz e impide que exista alta abundancia y riqueza. A diferencia, los brinzales presentan una sobrevivencia sobre $80 \%$, esta categoría se encuentra en un nivel intermedio de la vegetación, por lo cual tiende a ser más favorable en cuanto a disponibilidad de luz y otros recursos ambientales, el nivel de supresión disminuye. Por otra parte, existe mayor porcentaje de sobrevivencia en los latizales, representados sobre el $90 \%$, lo que permite deducir que las especies más desarrolladas tienen probabilidades altas de llegar a su etapa de madurez; es importante mencionar que los latizales tienden a ser más dominantes y las probabilidades de estar suprimidos es mínima debido a su estructura. 
Estos datos tienen relación con lo que mencionan Samper y Vallejo (2007) en un estudio sobre la estructura y dinámica de poblaciones de plantas en un bosque andino, en donde, determinan que la tasa de mortalidad es alta en función del tamaño de los individuos y clases diamétricas más pequeñas, al contrario que, si hay un tamaño grande y clases diamétricas altas, la mortalidad disminuye. Este análisis de sobrevivencia se asemeja a los resultados obtenidos este estudio que determinó que la sobrevivencia en cada categoría de regeneración natural plántula, brinzal y latizal se incrementa conforme avanza la edad de los individuos.

\subsection{INCREMENTO DIAMÉTRICO Y EN ALTURA POR CATEGORÍA DE REGENERACIÓN NATURAL BAJO EL DOSEL DE LA PLANTACIÓN DE PINUS RADIATA D. DON}

En la categoría de plántula, la especie dominante en crecimiento diamétrico y altura es Frangula granulosa (0,0012 m diámetro) y 0,14 m de altura con hábito de crecimiento arbóreo, siendo una especie de sucesión secundaria y gremio ecológico esciofita parcial; es una especie común de bosques maduros (Minga y Verdugo, 2016). La segunda especie dominante es arbustiva, Piper barbatum (0,0007 $\mathrm{m})$, con un incremento del diámetro menor a Frangula granulosa y con mayor crecimiento en altura Viburnum triphyllum $(0,07 \mathrm{~m})$ requiere alta presencia de luz para su desarrollo (Minga y Verdugo, 2016), lo cual es otorgada por las condiciones estructurales de la plantación forestal. Por otra parte, se evidenció alturas desde $0,14 \mathrm{~m}$ hasta $0,26 \mathrm{~m}$ y diámetros desde 0,0019 hasta $0,0031 \mathrm{~m}$ debido a que se encuentran en sus etapas iniciales de desarrollo.

En la categoría brinzal, con mayor incremento diamétrico destaca Clusia latipes $(0,005 \mathrm{~m})$, seguido por Solanum cf. cutervanum $(0,003 \mathrm{~m})$ de hábito arbustivo y heliófitas durables. Se debe mencionar que a diferencia de la categoría plántula Piper barbatum en su incremento diamétrico no es significativo en esta categoría. Por otra parte, el incremento en altura está dominado por especies arbóreas como Critoniopsis pycnantha $(0,55 \mathrm{~m})$ de sucesión secundaria de preferencia heliófita; Clethra fimbriata (0,54 $\mathrm{m})$ tiene sus particularidades como Minga y Verdugo (2016) menciona que, a pesar de ser una especie arbórea, en suelos pobres se mantiene como arbustivo. También se evidencia la presencia de especies que presentan un incremento menor en altura, tal es el caso de Oreopanax rosei (0,08 m), Myrsine sodiroana $(0,07 \mathrm{~m})$, Miconia lutescens $(0,05 \mathrm{~m})$ y Palicourea anceps $(0,03 \mathrm{~m})$ que se encuentran suprimidas por especies heliófitas dominantes.

La categoría de latizal demuestra que las especies en mayor estadio de desarrollo tienden a incrementar en diámetro, es el caso de Alnus acuminata $(0,009 \mathrm{~m})$ especie heliófita, Cedrela montana $(0,006 \mathrm{~m})$ esciofita, que por su etapa de madurez se encuentran como dominante. El incremento en altura está dominado por Alnus acuminata $(0,50 \mathrm{~m})$, Roupala monsperma $(0,38 \mathrm{~m})$, Frangula granulosa $(0,31$ 
m), son especies arbóreas de sucesión secundaria que se distinguen por requerir luz para su desarrollo. Los valores de altura en cada categoría son parecidos a los reportados por Franco, Betancur y Franco (2010) en un bosque andino, en donde registró individuos inferiores a 7,20 m de altura, de la misma manera Gálvez, Ordoñez y Bussmann (2003) registra individuos con altura media de 9,41 m a $2025 \mathrm{~m}$ s.n.m. y diámetro medio de 12,13 cm, superior a los de esta investigación. Sin embargo, Bussmann (2005) justifica que las especies tienden a disminuir su diámetro conforme se incrementa la altitud sobre el nivel de mar.

\subsection{ANÁLISIS DE VARIABLES AMBIENTALES BAJO EL DOSEL DE LA PLANTACIÓN DE PINUS RADIATA D. DON}

En la categoría de plántulas, existe correlación baja entre luminosidad y número de especies e individuos, esto debido a que se encuentran suprimidas por hojarasca, materia orgánica en descomposición y cobertura de dosel, las cuales permiten que haya un ingreso de luz promedio (31,40 \%), esto se asemeja a lo mencionado por León (2014), quien registró un ingreso de luz directa entre $30 \%$ - 50 \%; Jaramillo y Muñoz (2009) también reportan la importancia de luz en la regeneración natural que influye en la aparición de nuevas especies, lo cual justifica la poca presencia de individuos de plántulas bajo la plantación de Pinus radiata.

La pendiente tiene escasa correlación sobre la diversidad de especies y abundancia, esto es comparable según Álvarez, Carriel y Villacis (2019) que indican que la inclinación del terreno no influye en la diversidad de especies. Además, la correlación de la profundidad del suelo horizonte $\mathrm{O}(1 \mathrm{~cm}$ a 3,50 $\mathrm{cm}$ ) en la diversidad y abundancia de las especies es negativa débil; es necesario conocer los componentes químicos de este horizonte para determinar la influencia en el desarrollo inicial de las plantas, fase donde es más exigente en nutrientes; los datos de profundidad son inferiores a lo reportado por Manzares, Torres y Navarro (2004) que registran un valor de $8 \mathrm{~cm}$ en plantaciones de pino.

En la categoría de brinzales la profundidad del suelo y pendiente presentan correlación débil. La luminosidad indica valores de correlación negativos moderadamente débiles con las especies e individuos. Se puede intuir que por el estado de desarrollo de las plantas tienden a depender de las condiciones físicas del suelo y la luminosidad pasa a ser un factor secundario; por consiguiente, los latizales son plantas más desarrolladas que presentan alta competencia, por ende, limitan el desarrollo de plántulas según lo sostiene Jadán et al. (2019). Tienen correlación con la pendiente semejante a lo reportado por Jirón (2017) donde la correlación $(\mathrm{r}=0,58)$ de la diversidad de regeneración natural es positiva con este factor.

Los latizales si se correlación con la luz moderadamente; la luz es un factor relevante para procesos de floración y fructificación y posterior contribuir a la regeneración de nuevos individuos, esto se asemeja 
al estudio de Alvarado y Muñoz (2017) donde la cobertura arbórea se correlacionó positivamente con la riqueza de vegetación leñosa; León (2014) también menciona la correlación positiva entre luminosidad y la diversidad bajo plantaciones. Además, Baruch, Johnson y Yerena (2016) recalca que la baja irradiación solar disminuye y restringe el desarrollo de posibles especies colonizadoras y comparado con este estudio se corrobora con la alta presencia de especies de latizales ya que son mayores receptores de luminosidad.

\section{CONCLUSIONES}

La composición florística de la regeneración natural de especies vegetales leñosas nativas bajo la plantación de Pinus radiata está representada por 24 especies, en su mayoría son arbóreas, y la mayor diversidad y abundancia se observa en la categoría de latizales.

Las especies de mayor representatividad en las tres categorías son: Viburnum triphyllum tiene hábito arbustivo y es típica de sucesión secundaria perteneciente al gremio ecológico esciofita parcial, ya que puede convivir entre espacios de sombra y disponibilidad de luz; Piper barbatum es heliófita efímera que aparecen en las primeras etapas de sucesión y presentan mediana duración de vida.; Frangula granulosa es una especie esciofita parcial que aparece en la segunda y tercera etapa de la sucesión secundaria formando parte de sotobosque o parcialmente dominante; Oreopanax rosei es una especie que aparece en las últimas etapas de sucesión, se comporta como esciofita parcial y forma parte del sotobosque o como especie dominante en competencia con las demás.

Mayor porcentaje de sobrevivencia, crecimiento en diámetro y altura se observa en los individuos latizales, seguido de brinzales y plántulas. Se observa mayor sobrevivencia de los individuos de Piper barbatum, Viburnum tripphyllum y Frangula granulosa, en las tres categorías, lo cual indica que presentan mayor tolerancia a las condiciones de hábitat que provee la plantación.

La pendiente y profundidad del suelo del horizonte $\mathrm{O}$ no demuestran influencia significativa, la luminosidad juega un papel significativo en las tres categorías, esto debido a que es importante en el desarrollo de los gremios ecológicos de las especies, ya que la mayoría de especies presentes tienen un comportamiento de heliófitas efímeras y esciofitas parciales.

La regeneración natural de las especies observadas sugiere que es factible la recuperación de la vegetación natural, que por la diversidad y abundancia de especies en las diferentes categorías, se proyecta a futuro un bosque natural en caso de la eliminación de la plantación forestal.

\section{AGRADECIMIENTOS}

A la Universidad Nacional de Loja y al Equipo de investigación que conforma el Proyecto Procesos ecológicos de la vegetación en el Parque Universitario “Francisco Vivar Castro", Universidad Nacional 


\section{CONTRIBUCIÓN DE AUTORES}

ZAM, dirigió el proyecto de investigación, redacto y corrigió el manuscrito; LGN colecto la información de campo, sistematizo y analizó la información; NJD y JPT apoyaron en la identificación de las especies botánicas y en el análisis de la información. 


\section{BIBLIOGRAFÍA}

Aguirre Mendoza, Z., Celi Delgado, H., \& Herrera Herrera, C. (2018). Estructura y composición florística del bosque siempreverde montano bajo de la parroquia San Andrés, cantón Chinchipe, provincia de Zamora Chinchipe, Ecuador. Arnaldoa, 25(3), 923-938.

Aguirre Mendoza, Z., Díaz Ordoñez, E., Muñoz Chamba, J., y Muñoz Chamba, L. (2019). Sucesión natural bajo plantaciones de Pinus radiata D. Don (Pinaceae) y Eucalyptus globulus Labill. (Myrtaceae), en el sur del Ecuador. Arnaldoa, 26(3), 943-964.

Aguirre-Mendoza Z., C. Yaguana y T. Gaona (2016). Parque universitario de educación ambiental y recreación Ing. Francisco Vivar Castro. Universidad Nacional de Loja, Loja, Ecuador.

Alvarado, A y Muñoz, L. (2017). Evaluación de la regeneración natural y su relación con la altitud y cobertura de dosel en plantaciones no manejadas de Pinus patula en zonas alto andinas, en la provincia del Azuay (Tesis de grado, Universidad de Cuenca), Cuenca.

Álvarez, C., Carriel, M. R. A., y Villacís, J. (2019). Diversidad arbórea y su relación con la pendiente en plantaciones de Eucalyptus globulus. Boletín Técnico, Serie Zoológica, 13(12-13).

Añazco, M., Morales, M., Palacios, W., Vega, E., \& Cuesta, A. L. (2010). Sector Forestal Ecuatoriano: propuestas para una gestión forestal sostenible (No. 634.9209866 S446).

Baruch, Z., Johnson, E., y Yerena, E. (2016). What deters plant colonization in a tropical pine plantation?. Revista de Biologia Tropical, 64(2), 461-471.

Bussmann, R. W. (2005). Bosques andinos del sur de Ecuador, clasificación, regeneración y uso. Revista peruana de Biología, 12(2), 203-216.

Caranqui, J. (2017). Análisis de la regeneración natural después de la explotación de pino en el páramo de Tamboloma.

Carrere, R. (2005). Pinos y eucaliptos en Ecuador: símbolos de un modelo destructivo. World Rainforest Movement, 1-12.

Cavelier, J., y Santos, C. (1999). Efectos de plantaciones abandonadas de especies exóticas y nativas sobre la regeneración natural de un bosque montano en Colombia. Revista de Biología Tropical, 47(4), 775784.

Cruz, A. (2011). Efecto de la pendiente en la estructura de un bosque montano pluvial, noreste del área natural de manejo integrado Apolobamba (Doctoral dissertation).

Franco, M., Betancur, J., y Franco, P. (2010). Diversidad florística y estructura de remanentes de bosque andino en la zona de amortiguación del Parque Nacional Natural los Nevados, Cordillera Central Colombiana. Caldasia, 32(1).

Gálvez, J. R., Ordoñez, O. R., \& Bussmann, R. W. (2003). Estructura del bosque montano perturbado y no-perturbado en el Sur de Ecuador Structure of disturbed and undisturbed mountain forests in Southern Ecuador. Lyonia, 3, 83-98 
Jadán, O., Cedillo, H., Pillacela, P., Guallpa, D., Gordillo, A., Zea, P., ... \& Vaca, C. (2019). Regeneración de Pinus patula (Pinaceae) en ecosistemas naturales y plantaciones, en un gradiente altitudinal andino, Azuay, Ecuador. Revista de Biología Tropical, 67(1).

Jaramillo, L., y Muñoz, L. (2009). Evaluación de la regeneración natural de especies forestales del bosque tropical de montaña en la Estación Científica San Francisco bajo diferentes intensidades de raleo selectivo. Tesis de grado, Universidad Nacional de Loja, Loja. Ecuador.

Jirón, J. (2017). Análisis de la diversidad de la regeneración natural y su relación con variables biofísicas en la Reserva Silvestre Privada Quelantaro, Managua, 2015-2016 (Doctoral dissertation, Universidad Nacional Agraria).

León, K. (2014). Evaluación de la influencia de la luz en la regeneración natural de especies leñosas bajo plantaciones de pino (Pinus patula) y rodales naturales de aliso (Alnus acuminata) en bosques montanos de la Región Sur del Ecuador. (Tesis Ingeniería, Universidad Nacional de Loja). Loja, Ecuador.

Mejía, M y Barón, M. (2019). Evaluación de los aportes de los árboles de sombra a la avifauna asociada a cultivos de cacao en Santa María, Boyacá.

Minga Ochoa, D., \& Verdugo Navas, A. (2016). Árboles y arbustos de los ríos de Cuenca. Recuperado de http://dspace.uazuay.edu.ec/handle/datos/8784

Mostacedo, B., \& Fredericksen, T. (2000). Manual de métodos básicos de muestreo y análisis en ecología vegetal.

Orozco L., y C. Brumer. (2002). Inventarios forestales para bosques latifoliados en América Central. Centro Agronómico Tropical de Investigación y Enseñanza. Turrialba, Costa Rica.

Samper, C., \& Vallejo, M. I. (2007). Estructura y dinámica de poblaciones de plantas en un bosque andino. Revista Academia Colombiana de Ciencias, 31(118), 57-68.

Vásquez, E. (1995). Plan de Manejo Prototipo para Bosques Plantados de Pino. Quito.

Voss, O., Aguirre, N., \& Hofstede, R. (2001). Sistemas Forestales Integrales para l1040a Sierra del Ecuador. Editorial Abya Yala. 